\title{
Review \\ The Role of De Novo Variants in Patients with Congenital Diaphragmatic Hernia
}

\author{
Charlotte Bendixen ${ }^{1, *(1)}$ and Heiko Reutter ${ }^{2,3}$ (D) \\ 1 Unit of Paediatric Surgery, Department of General, Visceral, Vascular and Thoracic Surgery, \\ University Hospital Bonn, 53127 Bonn, Germany \\ 2 Institute of Human Genetics, University Hospital of Bonn, 53127 Bonn, Germany; reutter@uni-bonn.de \\ 3 Division of Neonatology and Paediatric Intensive Care, Department of Pediatrics and Adolescent Medicine, \\ University Hospital Erlangen, 91054 Erlangen, Germany \\ * Correspondence: charlotte.bendixen@ukbonn.de
}

check for updates

Citation: Bendixen, C.; Reutter, H. The Role of De Novo Variants in Patients with Congenital Diaphragmatic Hernia. Genes 2021, 12,1405. https://doi.org/10.3390/ genes12091405

Academic Editor: Diego Centonze

Received: 31 July 2021

Accepted: 8 September 2021

Published: 11 September 2021

Publisher's Note: MDPI stays neutral with regard to jurisdictional claims in published maps and institutional affiliations.

Copyright: (C) 2021 by the authors. Licensee MDPI, Basel, Switzerland. This article is an open access article distributed under the terms and conditions of the Creative Commons Attribution (CC BY) license (https:/ / creativecommons.org/licenses/by/ $4.0 /)$.

\begin{abstract}
The genetic etiology of congenital diaphragmatic hernia ( $\mathrm{CDH})$, a common and severe birth defect, is still incompletely understood. Chromosomal aneuploidies, copy number variations (CNVs), and variants in a large panel of $\mathrm{CDH}$-associated genes, both de novo and inherited, have been described. Due to impaired reproductive fitness, especially of syndromic CDH patients, and still significant mortality rates, the contribution of de novo variants to the genetic background of $\mathrm{CDH}$ is assumed to be high. This assumption is supported by the relatively low recurrence rate among siblings. Advantages in high-throughput genome-wide genotyping and sequencing methods have recently facilitated the detection of de novo variants in $\mathrm{CDH}$. This review gives an overview of the known de novo disease-causing variants in $\mathrm{CDH}$ patients.
\end{abstract}

Keywords: congenital diaphragmatic hernia; de novo variants; impaired reproductive fitness; mortality

\section{Introduction}

Congenital diaphragmatic hernia $(\mathrm{CDH})$ is a relatively common birth defect reported to affect 2-3 per 10,000 live births [1]. Due to a high early neonatal and prenatal mortality, the hidden prevalence might be even higher [2]. The term CDH comprises a variety of defects in the diaphragm, ranging from diaphragmatic eventration to localized defects of variable size and locations to diaphragmatic agenesis. The most common type is the so-called "Bochdalek hernia" (dorsolateral) on the left side. CDH leads to herniation of abdominal viscera into the thorax during early embryonic development. Newborn patients typically present with respiratory distress which is, in short, due to hypoplasia of the lungs accompanied by abnormal structure of pulmonary vessels and alveolar septa, and pulmonary hypertension. Advancements in the prenatal diagnosis and postnatal management of $\mathrm{CDH}$ have led to reduced but still high mortality rates of $20-30 \%$ [3,4]. Surviving patients often exhibit significant long-term morbidity [5].

The etiology of $\mathrm{CDH}$ is incompletely understood. It is suggested that both genetic and environmental factors contribute to $\mathrm{CDH}$, and although associations with different environmental factors have been described, no finding could be replicated to date [6]. From a medical genetics point of view, about $40 \%$ of $\mathrm{CDH}$ patients present syndromic. These patients present with additional anomalies of other organ systems ("non-isolated"), mostly cardiac defects, malformations of the central nervous system, urinary tract, and gastrointestinal system [7]. In these cases, a genetic diagnosis can be established more likely than in cases of isolated or non-syndromic $\mathrm{CDH}$. Overall, in about $30 \%$ of $\mathrm{CDH}$ patients disease-causing genetic aberrations can be identified by chromosomal analysis, molecular karyotyping, and exome/or genome sequencing. Here, it has been shown that about $6 \%$ of $\mathrm{CDH}$ patients present with chromosomal imbalances detectable by routine chromosomal analysis or molecular karyotyping [8]. Earlier reports describe detection rates of up to 
10\% [9]. Using a customized array comparative genomic hybridization assay, Zhu et al. reported likely causative CNVs in $13 \%$ of a mixed CDH cohort [10]. An additional 3-10\% of patients present with known monogenic syndromes. More recent sequencing studies have identified de novo damaging variants in known and novel CDH-associated genes in $10-30 \%$ of $\mathrm{CDH}$ patients [11-16]. Furthermore, is has been shown that the presence of a likely damaging de novo variant in a patient is associated with higher mortality and overall worse clinical outcome [17].

To establish a genetic diagnosis is increasingly important for affected families to provide proper counseling, especially as more $\mathrm{CDH}$ survivors reach reproductive age. This review focuses on the role of de novo events in $\mathrm{CDH}$ patients.

\section{Known Genetic Factors}

\subsection{Associated Microscopic and Submicroscopic Anomalies}

Except for the theoretical possibility of a trisomy 21 due to parental balanced translocation of chromosome 21 (not reported/investigated by most papers), all aneuploidies associated with $\mathrm{CDH}$ to date have been described to occur de novo. Aneuploidies (rarely) associated with $\mathrm{CDH}$ include trisomy 13, 18, 21, and triple X [17]. Furthermore it has been described in females with 45,X karyotype [18]. More frequently CDH has been described in patients with mosaic tetrasomy 12p (Pallister-Killian syndrome) [19], which always occurs de novo.

Other frequently detected CNVs include 15q26 deletion [20], comprising the CDHassociated gene NR2F2 [21]; 8p23.1 deletion [22], comprising the CDH-associated gene ZFPM2 [23]; 11q23 duplication typically resulting from parental balanced translocations [24], and 1q41-42 deletion [25], which includes the CDH-associated genes HLX and DISP1 [26,27].

Less frequently described in association with CDH 4p16 deletions (Wolf-Hirschhorn syndrome) [28,29], comprising the CDH-associated gene FGFRL1 [30]; 22q11.2 deletion [31]; deletion and duplication of 17q12 [32,33], and 1q12 duplication [34]. Very rare CNVs in $\mathrm{CDH}$ patients have been described and comprehensively been reviewed by Wynn et al. [18].

Among the CNVs found in $\mathrm{CDH}$ patients are, as expected, many de novo events. Other $\mathrm{CNV}$ s are caused by unbalanced translocations from a parental balanced translocation. Few CNVs are reported to be inherited [32,35]. The genome-wide de novo CNV rate in general is estimated to be $0.5-3 \%$ [36,37], about 2-12 times lower than the rate of de novo $\mathrm{CNVs}$ in $\mathrm{CDH}$ patients. CNVs are more likely to be detected in non-isolated cases of $\mathrm{CDH}$ than in isolated cases [8] and in general, more deletions (with a pathomechanism of haploinsufficency for $\mathrm{CDH}$-associated genes) have been reported. Overall, de novo CNVs have been shown to be a major contributor to the formation of $\mathrm{CDH}$.

\subsection{De Novo Variants in Monogenic CDH Syndromes}

More than 20 syndromes with known genetic causes have been associated with the occurrence of $\mathrm{CDH}$. Among these are dominant, recessive, and X-linked inherited syndromes. de novo events commonly play a role in autosomal dominant or X-linked syndromes. The rare occurrence of de novo events leading to a recessive $\mathrm{CDH}$-associated syndrome is described for Cutis laxa Type 1C [38]. Some well-known monogenic syndromes caused by de novo events and featuring CDH are Cornelia de Lange syndrome (NIPBL) [39,40]; Craniofrontonasal syndrome (EFNB1) [41]; Focal dermal hypoplasia (PORCN) [42]; and Kabuki syndrome (KMT2D; MLL2) [14,43,44]. A full list of monogenic syndromes in which de novo events are reported is provided in Table 1 . It has to be noted that for many described variants in other CDH-related autosomal dominant inherited syndromes, the inheritance pattern is not investigated or reported, but appears to be likely dominant de novo. 
Table 1. Monogenic syndromes with associated CDH caused by de novo events.

\begin{tabular}{|c|c|c|c|c|c|c|}
\hline Syndrome & OMIM & Gene & $\begin{array}{l}\text { Chromosomal } \\
\text { Location }\end{array}$ & $\begin{array}{c}\text { Genomic } \\
\text { Coordinates } \\
(\text { GRCh38/hg38) }\end{array}$ & Additional Malformations & References \\
\hline PDAC syndrome & \#615524 & $R A R B$ & $3 p 24.3$ & $\begin{array}{c}\text { chr3: } 25,428,263- \\
25,597,932\end{array}$ & $\begin{array}{l}\text { Micro-/Anophtalmia, } \\
\text { pulmonary hypoplasia, } \\
\text { cardiac abnormalities }\end{array}$ & [45] \\
\hline $\begin{array}{l}\text { Cornelia de } \\
\text { Lange syndrome }\end{array}$ & $\# 122470$ & NIPBL & $5 p 13.2$ & $\begin{array}{l}\text { chr5: } 36,876,769- \\
\quad 37,066,413\end{array}$ & $\begin{array}{l}\text { Hypertelorism, synophrys, } \\
\text { low anterior hairline, upper } \\
\text { limb malformations }\end{array}$ & {$[40,46,47]$} \\
\hline $\begin{array}{l}\text { Coffin-Siris } \\
\text { syndrome }\end{array}$ & $\begin{array}{l}\# 135900 \\
\# 614609\end{array}$ & $\begin{array}{c}\text { ARID1B, } \\
\text { SMARCA4 }\end{array}$ & $6 q 25.3$ & $\begin{array}{c}\text { chr6: } 156,776,020- \\
157,210,779 \\
\text { chr19: } 10,961,001- \\
11,062,256\end{array}$ & $\begin{array}{l}\text { Growth retardation, long } \\
\text { eyelashes, frequent } \\
\text { respiratory tract infections, } \\
\text { hypotonia, developmental } \\
\text { delay }\end{array}$ & {$[14,48]$} \\
\hline $\begin{array}{l}\text { Congenital heart } \\
\text { defects and } \\
\text { skeletal } \\
\text { malformations } \\
\text { syndrome } \\
\text { (CHDSKM) }\end{array}$ & \#617602 & $A B L 1$ & $9 q 34.12$ & $\begin{array}{c}\text { chr9: } 130,713,016- \\
130,885,683\end{array}$ & $\begin{array}{l}\text { Dysmorphic facial features, } \\
\text { congenital heart disease, } \\
\text { skeletal abnormalities, joint } \\
\text { laxity, failure to thrive, } \\
\text { gastrointestinal problems, } \\
\text { male genital anomalies }\end{array}$ & {$[14,49]$} \\
\hline Apert syndrome & \#101200 & FGFR2 & $10 q 26.13$ & $\begin{array}{c}\text { chr10: } \\
121,479,857- \\
121,598,403\end{array}$ & $\begin{array}{l}\text { Acrocephaly, micrognathia, } \\
\text { limb malformations }\end{array}$ & {$[50]$} \\
\hline $\begin{array}{l}\text { Denys-Drash } \\
\text { syndrome, } \\
\text { Meacham } \\
\text { syndrome }\end{array}$ & $\begin{array}{l}\# 194080 \\
\# 608978\end{array}$ & WT1 & $11 \mathrm{p} 13$ & $\begin{array}{c}\text { chr11: } 32,389,058- \\
32,435,360\end{array}$ & $\begin{array}{c}\text { Male } \\
\text { pseudohermaphroditism, } \\
\text { cardiac abnormalities }\end{array}$ & {$[51,52]$} \\
\hline Kabuki syndrome & \#147920 & $K M T 2 D$ & $12 q 13.12$ & $\begin{array}{c}\text { chr12: } 49,018,978- \\
49,060,794\end{array}$ & $\begin{array}{l}\text { Mental retardation, short } \\
\text { stature, eversion of eyelids, } \\
\text { finger pads }\end{array}$ & {$[14,43,44,53]$} \\
\hline $\begin{array}{l}\text { Marfan syndrome } \\
\text { Type } 1\end{array}$ & \#154700 & FBN1 & $15 q 21.1$ & $\begin{array}{c}\text { chr15: } 48,408,313- \\
48,645,709\end{array}$ & $\begin{array}{l}\text { Congenital contractures, } \\
\text { arachnodactyly, aortic } \\
\text { dilatation, cardiac valve } \\
\text { insufficiency }\end{array}$ & {$[14,54]$} \\
\hline $\begin{array}{l}\text { Geleophysic } \\
\text { dysplasia } 2\end{array}$ & \#614185 & FBN1 & $15 q 21.1$ & $\begin{array}{c}\text { chr15: } 48,408,313- \\
48,645,709\end{array}$ & $\begin{array}{l}\text { Short stature, cardiac } \\
\text { valvular thickening, skin } \\
\text { thickening, joint problems }\end{array}$ & [17] \\
\hline $\begin{array}{l}\text { Rubinstein-Taybi } \\
\text { syndrome } 2\end{array}$ & \#613684 & EP300 & $22 q 13.2$ & $\begin{array}{c}\text { chr22: } 41,092,592- \\
41,180,077\end{array}$ & $\begin{array}{c}\text { Failure to thrive, } \\
\text { cardiovascular } \\
\text { abnormalities, motor and } \\
\text { speech delays, dysmorphic } \\
\text { facial features }\end{array}$ & {$[14,55]$} \\
\hline $\begin{array}{l}\text { Focal dermal } \\
\text { hypoplasia }\end{array}$ & \#305600 & PORCN & Xp11.23 & $\begin{array}{c}\text { chrX: } 48,508,992- \\
48,520,808\end{array}$ & $\begin{array}{c}\text { Sparse hair, anophtalmia, } \\
\text { limb malformations, } \\
\text { Pentalogy of Cantrell }\end{array}$ & [42] \\
\hline $\begin{array}{l}\text { Craniofrontonasal } \\
\text { syndrome }\end{array}$ & \#304110 & EFNB1 & Xq13.1 & $\begin{array}{l}\text { chrX: } 68,829,021- \\
\quad 68,842,160\end{array}$ & $\begin{array}{l}\text { Coronal craniosynostosis, } \\
\text { duplex thumb, partial } \\
\text { agenesis of corpus callosum }\end{array}$ & [41] \\
\hline
\end{tabular}

\subsection{De Novo Variants in Non-Isolated CDH}

Several genes harboring de novo variants in non-isolated $\mathrm{CDH}$ patients have been identified, most of them by whole exome (WES)/whole genome (WGS) sequencing techniques. Among these are some well-known CDH-associated genes. de novo variants in GATA4 have been described in non-isolated [17,22,56] and isolated CDH [57]. GATA4 is known to be associated with congenital heart defects in humans and is further supported by a mouse model [58]. It encodes a transcription factor that is part of the retinoic acid signaling pathway, which has been implicated in diaphragm development [59].

Repeatedly, non-isolated CDH patients were found to carry de novo variants in NR2F2 [16,17,21,57], an interaction partner of ZFPM2, a gene commonly affected by the 
deletion of 8p23.1 observed in CDH patients. The role of NR2F2 in diaphragm development is further supported by its expression pattern and a mouse model [60]. More recently, de novo variants in $M Y R F$, a membrane associated transcription factor, have been described in non-isolated $\mathrm{CDH}$ patients, also showing cardiac and genitourinary malformations [12,17,61-63].

Other genes with described de novo variants in non-isolated $\mathrm{CDH}$ patients are listed in Table 2. Clinical features of patients are available in Table S1. In very few genes, variants in more than one patient could be detected. This illustrates the heterogeneity of the genetic background of $\mathrm{CDH}$. The largest WES/WGS study on family trios could identify de novo likely gene-disrupting (LGD) or deleterious missense (D-mis) variants in $21 \%$ of nonisolated CDH cases [12]. Another family trio study also showed an increased burden of de novo D-mis and LGD variants in a mixed cohort of isolated and non-isolated CDH [13]. Recently a WES study established a genetic diagnosis in 28/76 (37\%) non-isolated CDH patients, of which 15/76 (20\%) were attributable to de novo variants [14]. These findings further strongly support a major role of de novo variants in $\mathrm{CDH}$.

Table 2. Genes with de novo variants in non-isolated $\mathrm{CDH}$ patients.

\begin{tabular}{|c|c|c|c|c|c|}
\hline Gene & $\begin{array}{l}\text { Chromosomal } \\
\text { Location }\end{array}$ & $\begin{array}{c}\text { Genomic } \\
\text { Coordinates } \\
\text { (GRCh38/hg38) }\end{array}$ & $\begin{array}{c}\text { Number of } \\
\text { Patients with de } \\
\text { novo Variants }\end{array}$ & References & $\begin{array}{l}\text { Design/Method } \\
\text { of Studies }\end{array}$ \\
\hline$P R K A C B$ & $1 \mathrm{p} 31.1$ & $\begin{array}{c}\text { chr1: } 84,078,062- \\
84,238,498\end{array}$ & 1 & [14] & trio WES \\
\hline SLC5A9 & 1p33 & $\begin{array}{c}\text { chr1: } 48,222,716- \\
48,248,638\end{array}$ & 1 & [14] & trio WES \\
\hline ZNF362 & 1p35.1 & $\begin{array}{c}\text { chr1: } 33,256,492- \\
33,300,719\end{array}$ & 1 & [17] & trio WES/WGS \\
\hline HSPG2 & 1p36.12 & $\begin{array}{c}\text { chr1: } 21,822,244- \\
21,937,310\end{array}$ & $1^{\circ}$ & [17] & trio WES \\
\hline$U B A P 2 L$ & $1 \mathrm{q} 21.3$ & $\begin{array}{c}\text { chr1: } 154,220,955- \\
154,270,847\end{array}$ & 1 & [17] & trio WGS \\
\hline POGZ & $1 \mathrm{q} 21.3$ & $\begin{array}{c}\text { chr1: } 151,402,724- \\
151,459,494\end{array}$ & 1 & [12] & clinical WES \\
\hline DISP1 & $1 \mathrm{q} 41$ & $\begin{array}{c}\text { chr1: } 222,815,039- \\
223,005,995\end{array}$ & 1 & [27] & $\begin{array}{l}\text { targeted sanger } \\
\text { sequencing }\end{array}$ \\
\hline$I N H B B$ & $2 q 14.2$ & $\begin{array}{c}\text { chr2: } 120,346,136- \\
120,351,803\end{array}$ & 1 & [14] & trio WES \\
\hline TTC21B & $2 \mathrm{q} 24.3$ & $\begin{array}{c}\text { chr2: } 165,873,362- \\
165,953,776\end{array}$ & 1 & [17] & trio WGS \\
\hline ROBO1 & $3 p 12.3$ & $\begin{array}{c}\text { chr3: 78,598,688- } \\
79,019,015\end{array}$ & 1 & [17] & $\begin{array}{l}\text { targeted panel } \\
\text { sequencing }\end{array}$ \\
\hline FOXP1 & 3p13 & $\begin{array}{c}\text { chr3: } 70,954,708- \\
71,583,978\end{array}$ & 1 & [15] & clinical WES \\
\hline$R A F 1$ & $3 p 25.2$ & $\begin{array}{c}\text { chr3: } 12,583,601- \\
12,664,117\end{array}$ & 1 & [12] & trio WES/WGS \\
\hline FAT4 & $4 q 28.1$ & $\begin{array}{c}\text { chr4: } 125,314,955- \\
125,492,932\end{array}$ & 1 & [17] & trio WGS \\
\hline CDO1 & $5 q 22.3$ & $\begin{array}{c}\text { chr5: } 115,804,733- \\
115,816,659\end{array}$ & 1 & [14] & trio WES \\
\hline FOXP4 & $6 \mathrm{p} 21.1$ & $\begin{array}{c}\text { chr6: } 41,546,426- \\
41,602,384\end{array}$ & 1 & [12] & trio WES/WGS \\
\hline PTPN12 & $7 q 11.23$ & $\begin{array}{c}\text { chr7: } 77,537,295- \\
77,640,069\end{array}$ & 1 & [14] & trio WES \\
\hline
\end{tabular}


Table 2. Cont.

\begin{tabular}{|c|c|c|c|c|c|}
\hline Gene & $\begin{array}{l}\text { Chromosomal } \\
\text { Location }\end{array}$ & $\begin{array}{c}\text { Genomic } \\
\text { Coordinates } \\
\text { (GRCh38/hg38) }\end{array}$ & $\begin{array}{l}\text { Number of } \\
\text { Patients with } d e \\
\text { novo Variants }\end{array}$ & References & $\begin{array}{l}\text { Design/Method } \\
\text { of Studies }\end{array}$ \\
\hline$B R A F$ & $7 q 34$ & $\begin{array}{c}\text { chr7: } 140,719,327- \\
140,924,810\end{array}$ & 1 & [12] & trio WES/WGS \\
\hline GATA4 & $8 \mathrm{p} 23.1$ & $\begin{array}{c}\text { chr8: } 11,704,202- \\
11,760,002\end{array}$ & 3 & {$[17,22,56]$} & $\begin{array}{c}\text { targeted sanger } \\
\text { sequencing, trio WGS }\end{array}$ \\
\hline EYA1 & $8 \mathrm{q} 13.3$ & $\begin{array}{c}\text { chr8: } 71,197,511- \\
\quad 71,548,061\end{array}$ & 1 & {$[11,57]$} & $\begin{array}{l}\text { WES, targeted panel } \\
\text { sequencing }\end{array}$ \\
\hline TLN1 & 9p13.3 & $\begin{array}{c}\text { chr9: } 35,696,948- \\
35,732,195\end{array}$ & $1^{\circ}$ & [17] & trio WES \\
\hline PLPP6 & $9 \mathrm{p} 24.1$ & $\begin{array}{c}\text { chr9: } \\
4,662,294-4,665,258\end{array}$ & 1 & [14] & trio WES \\
\hline NOTCH1 & $9 q 34.3$ & $\begin{array}{c}\text { chr9: } 136,494,433- \\
136,546,048\end{array}$ & 1 & [17] & trio WGS \\
\hline CTR9 & 11 p15.3 & $\begin{array}{c}\text { chr11: } 10,751,246- \\
10,779,746\end{array}$ & $1 *$ & [16] & trio WES \\
\hline$M Y R F$ & $11 q 12.2$ & $\begin{array}{c}\text { chr11: } 61,752,636- \\
\quad 61,788,518\end{array}$ & 11 & {$[12,17,61-63]$} & $\begin{array}{c}\text { trio WES/WGS, } \\
\text { clinical WES, trio WGS }\end{array}$ \\
\hline PTPN11 & $12 q 24.13$ & $\begin{array}{c}\text { chr12: } 112,419,112- \\
112,504,764\end{array}$ & 1 & [12] & trio WES/WGS \\
\hline HNRNPC & $14 q 11.2$ & $\begin{array}{c}\text { chr14: } 21,210,613- \\
21,269,421\end{array}$ & 1 & [17] & trio WGS \\
\hline$B M P 4$ & $14 q 22.2$ & $\begin{array}{c}\text { chr14: } 53,949,736- \\
53,956,825\end{array}$ & 1 & [64] & $\begin{array}{l}\text { targeted sanger } \\
\text { sequencing }\end{array}$ \\
\hline$D L S T$ & $14 \mathrm{q} 24.3$ & $\begin{array}{c}\text { chr14: } 74,881,916- \\
74,903,743\end{array}$ & 1 & [14] & trio WES \\
\hline TCF12 & $15 q 21.3$ & $\begin{array}{c}\text { chr15: } 56,918,644- \\
57,289,853\end{array}$ & 1 & [15] & clinical WES \\
\hline SIN3A & $15 q 24.2$ & $\begin{array}{c}\text { chr15: } 75,370,933- \\
75,455,783\end{array}$ & 1 & [14] & trio WES \\
\hline$N R 2 F 2$ & $15 q 26.2$ & $\begin{array}{c}\text { chr15: } 96,330,700- \\
96,340,258\end{array}$ & 4 & {$[16,17,21,57,65]$} & $\begin{array}{c}\text { clinical WES, targeted } \\
\text { panel sequencing, trio } \\
\text { WES, trio WGS }\end{array}$ \\
\hline TRAF7 & $16 \mathrm{p} 13.3$ & $\begin{array}{c}\operatorname{chr} 16: \\
2,155,782-2,178,129\end{array}$ & 1 & [15] & clinical WES \\
\hline ANKRD11 & $16 \mathrm{q} 24.3$ & $\begin{array}{c}\text { chr16: } 89,285,175- \\
89,490,318\end{array}$ & 1 & [17] & trio WGS \\
\hline MYH10 & 17p13.1 & $\begin{array}{c}\operatorname{chr} 17: \\
8,474,207-8,630,761\end{array}$ & 1 & [66] & clinical WES \\
\hline TP53 & $17 \mathrm{p} 13.1$ & $\begin{array}{c}\operatorname{chr} 17: \\
7,668,421-7,687,490\end{array}$ & 1 * & [16] & trio WES \\
\hline$N L K$ & $17 \mathrm{q} 11.2$ & $\begin{array}{c}\text { chr17: } 28,042,677- \\
28,196,381\end{array}$ & 1 & [17] & trio WGS \\
\hline FZD2 & $17 q 21.31$ & $\begin{array}{c}\text { chr17: } 44,557,484- \\
44,561,262\end{array}$ & 1 & [32] & aCGH \\
\hline ATXN7L3 & $17 q 21.31$ & $\begin{array}{c}\text { chr17: } 44,191,805- \\
44,198,070\end{array}$ & 1 & [17] & trio WGS \\
\hline$A L Y R E F$ & $17 q 25.3$ & $\begin{array}{c}\text { chr17: } 81,887,835- \\
81,891,586\end{array}$ & 1 & [12] & trio WES/WGS \\
\hline
\end{tabular}


Table 2. Cont.

\begin{tabular}{|c|c|c|c|c|c|}
\hline Gene & $\begin{array}{l}\text { Chromosomal } \\
\text { Location }\end{array}$ & $\begin{array}{c}\text { Genomic } \\
\text { Coordinates } \\
\text { (GRCh38/hg38) }\end{array}$ & $\begin{array}{l}\text { Number of } \\
\text { Patients with de } \\
\text { novo Variants }\end{array}$ & References & $\begin{array}{c}\text { Design/Method } \\
\text { of Studies }\end{array}$ \\
\hline GATA6 & $18 q 11.2$ & $\begin{array}{c}\text { chr18: } 22,169,589- \\
22,202,528\end{array}$ & 1 & [67] & trio WES \\
\hline NACC1 & 19p13.13 & $\begin{array}{c}\text { chr19: } 13,118,264- \\
13,141,147\end{array}$ & 1 & [12] & trio WES/WGS \\
\hline LONP1 & $19 \mathrm{p} 13.3$ & $\begin{array}{c}\operatorname{chr} 19: \\
5,691,835-5,720,572\end{array}$ & 1 & [14] & trio WES \\
\hline LTBP4 & $19 q 13.2$ & $\begin{array}{c}\text { chr19: } 40,601,369- \\
40,629,818\end{array}$ & 1 & [38] & $\begin{array}{l}\text { targeted sanger } \\
\text { sequencing }\end{array}$ \\
\hline $\mathrm{ZC} 3 \mathrm{H} 4$ & $19 q 13.32$ & $\begin{array}{c}\text { chr19: } 47,064,187- \\
47,113,776\end{array}$ & 1 & [12] & trio WES/WGS \\
\hline$P C N A$ & $20 \mathrm{p} 12.3$ & $\begin{array}{c}\operatorname{chr} 20: \\
5,114,953-5,126,626\end{array}$ & 1 & [12] & trio WES/WGS \\
\hline EPB41L1 & $20 \mathrm{q} 11.23$ & $\begin{array}{c}\text { chr20: } 36,092,712- \\
36,230,343\end{array}$ & 1 & [12] & trio WES/WGS \\
\hline ARFGEF2 & $20 \mathrm{q} 13.13$ & $\begin{array}{c}\text { chr20: } 48,921,711- \\
49,036,693\end{array}$ & 1 & [14] & trio WES \\
\hline$A D N P$ & $20 \mathrm{q} 13.13$ & $\begin{array}{c}\text { chr20: } 50,888,918- \\
50,931,437\end{array}$ & 1 & [17] & trio WGS \\
\hline SCAF4 & $21 q 22.11$ & $\begin{array}{c}\text { chr21: } 31,671,000- \\
31,732,118\end{array}$ & 1 & [17] & trio WGS \\
\hline DDX3X & Xp11.4 & $\begin{array}{c}\text { chrX: } 41,333,348- \\
41,350,287\end{array}$ & 1 & [15] & clinical WES \\
\hline USP9X & Xp11.4 & $\begin{array}{c}\text { chrX: } 41,085,445- \\
\quad 41,236,579\end{array}$ & $1^{\circ}$ & [17] & trio WES/WGS \\
\hline CLCN4 & Xp22.2 & $\begin{array}{c}\text { chrX: } 10,156,975- \\
10,237,660\end{array}$ & 1 & [14] & trio WES \\
\hline HCCS & Xp22.2 & $\begin{array}{c}\text { chrX: } 11,111,301- \\
11,123,078\end{array}$ & 1 & [15] & clinical WES \\
\hline STAG2 & Xq25 & $\begin{array}{c}\text { chrX: } 123,961,314- \\
124,102,656\end{array}$ & 1 & [14] & trio WES \\
\hline
\end{tabular}

* Variants reported in the same patient, additionally de novo CNV deletion 8p23. ${ }^{\circ}$ Variants reported in the same patient.

\subsection{De Novo Variants in Isolated $C D H$}

In patients with isolated $\mathrm{CDH}$ a genetic cause is less likely to be established by current genotyping or sequencing techniques. The above-mentioned study on case-parent-trios could identify de novo likely gene-disrupting or deleterious missense variants in only $12 \%$ of isolated $\mathrm{CDH}$ cases [12]. Among the described de novo variants in isolated $\mathrm{CDH}$ are variants in the already mentioned genes ZFPM2 [12,23,68], GATA4 [57], and PTPN11 [12,16,17]. As in non-isolated $\mathrm{CDH}$, variants in very few genes could be implicated in more than one patient. A list of genes with de novo variants in isolated $\mathrm{CDH}$ is provided in Table 3. Notably, some genes are reported to carry de novo variants in non-isolated and isolated $\mathrm{CDH}$ patients. 
Table 3. Genes with de novo variants in isolated $\mathrm{CDH}$ patients.

\begin{tabular}{|c|c|c|c|c|c|}
\hline Gene & $\begin{array}{l}\text { Chromosomal } \\
\text { Location }\end{array}$ & $\begin{array}{l}\text { Genomic Coordinates } \\
\text { (GRCh38/hg38) }\end{array}$ & $\begin{array}{l}\text { Number of Patients } \\
\text { with de novo Variants }\end{array}$ & References & $\begin{array}{l}\text { Design/Method } \\
\text { of Studies }\end{array}$ \\
\hline HSPG2 & $1 \mathrm{p} 36.12$ & chr1: $21,822,244-21,937,310$ & 2 & {$[13,14]$} & trio WES \\
\hline ATAD3A & $1 \mathrm{p} 36.33$ & chr1: $1,512,175-1,534,685$ & 1 & [12] & trio WES/WGS \\
\hline POGZ & $1 \mathrm{q} 21.3$ & chr1: $151,402,724-151,459,494$ & 1 & [12] & trio WES/WGS \\
\hline KDM5B & $1 \mathrm{q} 32.1$ & chr1: $202,724,495-202,808,421$ & 1 & [12] & trio WES/WGS \\
\hline ZBTB18 & $1 \mathrm{q} 44$ & chr1: $244,051,283-244,057,476$ & 1 & [12] & trio WES/WGS \\
\hline MYT1L & $2 p 25.3$ & chr2: $1,789,124-2,331,348$ & 1 & [12] & trio WES/WGS \\
\hline FOXP1 & $3 p 13$ & chr3: 70,954,708-71,583,978 & 1 & [12] & trio WES/WGS \\
\hline SRGAP3 & $3 p 25.3$ & chr3: $8,980,594-9,249,213$ & 1 & [12] & trio WES/WGS \\
\hline KPNA1 & $3 q 21.1$ & chr3: $122,421,902-122,514,939$ & 1 & [17] & trio WGS \\
\hline NAA15 & $4 \mathrm{q} 31.1$ & chr4: $139,301,505-139,391,384$ & 1 & [12] & trio WES/WGS \\
\hline SMO & $7 \mathrm{q} 32.1$ & chr7: $129,188,633-129,213,545$ & 1 & [12] & trio WES/WGS \\
\hline GATA4 & $8 \mathrm{p} 23.1$ & chr8: $11,704,202-11,760,002$ & 1 & [57] & $\begin{array}{l}\text { targeted panel } \\
\text { sequencing }\end{array}$ \\
\hline ZFPM2 & $8 \mathrm{q} 23.1$ & chr8: $105,318,438-105,804,539$ & 3 & {$[12,23,68]$} & $\begin{array}{c}\text { WES, trio WES/WGS, } \\
\text { targeted sanger } \\
\text { sequencing }\end{array}$ \\
\hline$E M X 2$ & $10 \mathrm{q} 26.11$ & chr10: $117,542,746-117,549,546$ & 1 & [12] & trio WES/WGS \\
\hline WT1 & $11 \mathrm{p} 13$ & chr11: $32,389,058-32,435,360$ & 3 & {$[12,16]$} & trio WES/WGS \\
\hline PTPN11 & $12 q 24.13$ & chr12: $112,419,112-112,504,764$ & 3 & {$[12,16,17]$} & trio WES/WGS \\
\hline MEIS2 & $15 q 14$ & chr15: 36,889,204-37,100,549 & 1 & [12] & trio WES/WGS \\
\hline TBX6 & $16 \mathrm{p} 11.2$ & chr16: 30,085,793-30,091,924 & 1 & [11] & WES \\
\hline CTCF & $16 \mathrm{q} 22.1$ & chr16: $67,562,467-67,639,176$ & 1 & [17] & trio WGS \\
\hline AP1G1 & $16 \mathrm{q} 22.2$ & chr16: $71,729,000-71,808,834$ & 1 & [12] & trio WES/WGS \\
\hline MYH10 & 17p13.1 & chr17: 8,474,207-8,630,761 & 1 & [17] & $\begin{array}{l}\text { targeted panel } \\
\text { sequencxing }\end{array}$ \\
\hline SRSF1 & $17 q 22$ & chr17: $58,000,919-58,007,246$ & 1 & [17] & trio WGS \\
\hline LONP1 & 19p13.3 & chr19: 5,691,835-5,720,572 & 2 & [17] & trio WGS \\
\hline CIC & 19q13.2 & chr19: 42,268,537-42,295,796 & 1 & [12] & trio WES/WGS \\
\hline LAMA5 & $20 q 13.33$ & chr20: $62,309,065-62,367,312$ & 1 & [12] & trio WES/WGS \\
\hline DIDO1 & $20 q 13.33$ & chr20: $62,877,738-62,937,952$ & 1 & [12] & trio WES/WGS \\
\hline HSD17B10 & Xp11.22 & chrX: 53,431,261-53,434,370 & 1 & [12] & trio WES/WGS \\
\hline FLNA & Xq28 & chrX: $154,348,529-154,371,283$ & 1 & [17] & trio WGS \\
\hline
\end{tabular}

\section{Discussion}

Based on the current knowledge, we have to assume that de novo events play a major role in $\mathrm{CDH}$ etiology. In up to $30 \%$ of $\mathrm{CDH}$ cases a genetic cause can be established, more often in non-isolated than in isolated CDH. For the estimation of the fraction of causal $\mathrm{CNVs}$ /variants being de novo, large family trio studies are needed. However, in these, often only de novo events are reported. By looking at subsets of two large CNV studies $[8,10]$ the fraction of causal CNVs being de novo can be estimated up to $80 \%$. Similarly, the fraction of causal variants being de novo could be estimated around 50\% [15]. However, these estimations are based on small sample sizes only. Most likely, the fraction of de novo events is currently underestimated due to restricted genetic testing for newborns with (especially sporadic isolated) $\mathrm{CDH}$ in clinical practice.

The contribution of de novo variants to a disease depends on several factors. (i) It is higher in sporadic than in familial diseases; (ii) it is higher when the impact on fitness of the disease is higher; (iii) it is higher in monogenic than in complex diseases [69]. On the other hand, the incidence of a disease caused by de novo events increases with (i) mutational 
target size; (ii) target mutability and (iii) paternal age at conception [69]. When conferring this to $\mathrm{CDH}, \mathrm{CDH}$ is a mostly sporadic disease with high impact on fitness with not fully understood genetics, but monogenic forms being reported. The mutational target size is most likely large due to the heterogeneity of $\mathrm{CDH}$. Paternal age at conception has not been reported to be a risk factor for $\mathrm{CDH}$.

A well-studied example of a condition with reduced reproductive fitness is developmental delay/intellectual disability (DD/ID). Here it could be shown that de novo variants account for $\sim 50 \%$ of the genetic background of DD/ID [70]. For CDH, a similar or even higher proportion can be hypothesized. Larger whole genome/whole exome sequencing studies on case-parent-trios will most likely reveal additional de novo variants. The pathogenicity of the many rare de novo variants reported in $\mathrm{CDH}$ patients could also be further supported by larger resequencing studies which would identify additional patients harboring the same variant.

Genetic counseling for affected families with the sporadic occurrence of non-syndromic $\mathrm{CDH}$ should however, imply the recurrence risk of about $1 \%$ in future pregnancies. This, however, changes accordingly, when a genetic diagnosis has been established. Regardless of the establishment of a genetic diagnosis, affected families should be referred to a prenatal medicine center during the first and second trimester of subsequent pregnancies.

\section{Conclusions}

Among rare and severe birth defects, $\mathrm{CDH}$ is one of the more common ones. The current knowledge on the genetics of $\mathrm{CDH}$ suggests that a substantial fraction of $\mathrm{CDH}$ is due to underlying genetic de novo events. However, it is conceivable that several common variants form a "risk haplotype" that predisposes to non-syndromic $\mathrm{CDH}$.

Supplementary Materials: The following are available online at https:/ /www.mdpi.com/article/10 .3390 /genes12091405/s1, Table S1: Additional clinical features of non-isolated CDH patients from Table 2.

Author Contributions: Writing—original draft preparation, C.B.; writing—review and editing, H.R. All authors have read and agreed to the published version of the manuscript.

Funding: C.B. is supported by BONFOR stipend O-112.0062.

Institutional Review Board Statement: Not applicable.

Informed Consent Statement: Not applicable.

Data Availability Statement: Not applicable.

Conflicts of Interest: The authors declare no conflict of interest.

\section{References}

1. McGivern, M.R.; Best, K.E.; Rankin, J.; Wellesley, D.; Greenlees, R.; Addor, M.C.; Arriola, L.; de Walle, H.; Barisic, I.; Beres, J.; et al. Epidemiology of congenital diaphragmatic hernia in Europe: A register-based study. Arch. Dis. Child. Fetal Neonatal Ed. 2015, 100, F137-44. [CrossRef]

2. Burgos, C.M.; Frenckner, B. Addressing the hidden mortality in CDH: A population-based study. J. Pediatr. Surg. 2017, 52, 522-525. [CrossRef]

3. van den Hout, L.; Reiss, I.; Felix, J.F.; Hop, W.C.; Lally, P.A.; Lally, K.P.; Tibboel, D. Congenital Diaphragmatic Hernia Study Group. Risk factors for chronic lung disease and mortality in newborns with congenital diaphragmatic hernia. Neonatology 2010, 98, 370-380. [CrossRef]

4. $\quad$ van den Hout, L.; Schaible, T.; Cohen-Overbeek, T.E.; Hop, W.; Siemer, J.; van de Ven, K.; Wessel, L.; Tibboel, D.; Reiss, I. Actual outcome in infants with congenital diaphragmatic hernia: The role of a standardized postnatal treatment protocol. Fetal Diagn. Ther. 2011, 29, 55-63. [CrossRef] [PubMed]

5. Chiu, P.P.; Ijsselstijn, H. Morbidity and long-term follow-up in CDH patients. Eur. J. Pediatr. Surg. 2012, 22, 384-392. [CrossRef] [PubMed]

6. Schulz, F.; Jenetzky, E.; Zwink, N.; Bendixen, C.; Kipfmueller, F.; Rafat, N.; Heydweiller, A.; Wessel, L.; Reutter, H.; Mueller, A.; et al. Parental risk factors for congenital diaphragmatic hernia-A large German case-control study. BMC Pediatr. 2021, $21,278$. [CrossRef] [PubMed] 
7. Pober, B.R. Overview of epidemiology, genetics, birth defects, and chromosome abnormalities associated with CDH. Am. J. Med. Genet. C Semin. Med. Genet. 2007, 145C, 158-171. [CrossRef]

8. Yu, L.; Wynn, J.; Ma, L.; Guha, S.; Mychaliska, G.B.; Crombleholme, T.M.; Azarow, K.S.; Lim, F.Y.; Chung, D.H.; Potoka, D.; et al. de novo copy number variants are associated with congenital diaphragmatic hernia. J. Med. Genet. 2012, 49, 650-659. [CrossRef] [PubMed]

9. Garne, E.; Haeusler, M.; Barisic, I.; Gjergja, R.; Stoll, C.; Clementi, M. Euroscan Study Group. Congenital diaphragmatic hernia: Evaluation of prenatal diagnosis in 20 European regions. Ultrasound Obstet. Gynecol. 2002, 19, 329-333. [CrossRef] [PubMed]

10. Zhu, Q.; High, F.A.; Zhang, C.; Cerveira, E.; Russell, M.K.; Longoni, M.; Joy, M.P.; Ryan, M.; Mil-Homens, A.; Bellfy, L.; et al. Systematic analysis of copy number variation associated with congenital diaphragmatic hernia. Proc. Natl. Acad. Sci. USA 2018, 115, 5247-5252. [CrossRef]

11. Longoni, M.; High, F.A.; Russell, M.K.; Kashani, A.; Tracy, A.A.; Coletti, C.M.; Hila, R.; Shamia, A.; Wells, J.; Ackerman, K.G.; et al. Molecular pathogenesis of congenital diaphragmatic hernia revealed by exome sequencing, developmental data, and bioinformatics. Proc. Natl. Acad. Sci. USA 2014, 111, 12450-12455. [CrossRef]

12. Qi, H.; Yu, L.; Zhou, X.; Wynn, J.; Zhao, H.; Guo, Y.; Zhu, N.; Kitaygorodsky, A.; Hernan, R.; Aspelund, G.; et al. de novo variants in congenital diaphragmatic hernia identify MYRF as a new syndrome and reveal genetic overlaps with other developmental disorders. PLoS Genet. 2018, 14, e1007822. [CrossRef]

13. Longoni, M.; High, F.A.; Qi, H.; Joy, M.P.; Hila, R.; Coletti, C.M.; Wynn, J.; Loscertales, M.; Shan, L.; Bult, C.J.; et al. Genome-wide enrichment of damaging de novo variants in patients with isolated and complex congenital diaphragmatic hernia. Hum. Genet. 2017, 136, 679-691. [CrossRef]

14. Yu, L.; Sawle, A.D.; Wynn, J.; Aspelund, G.; Stolar, C.J.; Arkovitz, M.S.; Potoka, D.; Azarow, K.S.; Mychaliska, G.B.; Shen, Y.; et al. Increased burden of de novo predicted deleterious variants in complex congenital diaphragmatic hernia. Hum. Mol. Genet. 2015, 24, 4764-4773. [CrossRef] [PubMed]

15. Scott, T.M.; Campbell, I.M.; Hernandez-Garcia, A.; Lalani, S.R.; Liu, P.; Shaw, C.A.; Rosenfeld, J.A.; Scott, D.A. Clinical exome sequencing data reveal high diagnostic yields for congenital diaphragmatic hernia plus $(\mathrm{CDH}+)$ and new phenotypic expansions involving CDH. J. Med. Genet. 2020. [CrossRef]

16. Schwab, M.E.; Dong, S.; Lianoglou, B.R.; Aguilar Lucero, A.F.; Schwartz, G.B.; Norton, M.E.; MacKenzie, T.C.; Sanders, S.J. Exome sequencing of fetuses with congenital diaphragmatic hernia supports a causal role for NR2F2, PTPN11, and WT1 variants. Am. J. Surg. 2021, 20. [CrossRef]

17. Qiao, L.; Wynn, J.; Yu, L.; Hernan, R.; Zhou, X.; Duron, V.; Aspelund, G.; Farkouh-Karoleski, C.; Zygumunt, A.; Krishnan, U.S.; et al. Likely damaging de novo variants in congenital diaphragmatic hernia patients are associated with worse clinical outcomes. Genet. Med. 2020, 22, 2020-2028. [CrossRef]

18. Wynn, J.; Yu, L.; Chung, W.K. Genetic causes of congenital diaphragmatic hernia. Semin. Fetal Neonatal. Med. 2014, 19, 324-330. [CrossRef] [PubMed]

19. Salzano, E.; Raible, S.E.; Kaur, M.; Wilkens, A.; Sperti, G.; Tilton, R.K.; Bettini, L.R.; Rocca, A.; Cocchi, G.; Selicorni, A.; et al. Prenatal profile of Pallister-Killian syndrome: Retrospective analysis of 114 pregnancies, literature review and approach to prenatal diagnosis. Am. J. Med. Genet. A 2018, 176, 2575-2586. [CrossRef] [PubMed]

20. Mosca, A.L.; Pinson, L.; Andrieux, J.; Copin, H.; Bigi, N.; Puechberty, J.; Sarda, P.; Receveur, A.; Sevestre, H.; Pigeonnat, S.; et al. Refining the critical region for congenital diaphragmatic hernia on chromosome $15 \mathrm{q} 26$ from the study of four fetuses. Prenat Diagn. 2011, 31, 912-914. [CrossRef]

21. High, F.A.; Bhayani, P.; Wilson, J.M.; Bult, C.J.; Donahoe, P.K.; Longoni, M. de novo frameshift mutation in COUP-TFII (NR2F2) in human congenital diaphragmatic hernia. Am. J. Med. Genet. A 2016, 170, 2457-2461. [CrossRef]

22. Longoni, M.; Lage, K.; Russell, M.K.; Loscertales, M.; Abdul-Rahman, O.A.; Baynam, G.; Bleyl, S.B.; Brady, P.D.; Breckpot, J.; Chen, C.P.; et al. Congenital diaphragmatic hernia interval on chromosome 8p23.1 characterized by genetics and protein interaction networks. Am. J. Med. Genet. A 2012, 158A, 3148-3158. [CrossRef] [PubMed]

23. Longoni, M.; Russell, M.K.; High, F.A.; Darvishi, K.; Maalouf, F.I.; Kashani, A.; Tracy, A.A.; Coletti, C.M.; Loscertales, M.; Lage, K.; et al. Prevalence and penetrance of ZFPM2 mutations and deletions causing congenital diaphragmatic hernia. Clin. Genet. 2015, 87, 362-367. [CrossRef]

24. Klaassens, M.; Scott, D.A.; van Dooren, M.; Hochstenbach, R.; Eussen, H.J.; Cai, W.W.; Galjaard, R.J.; Wouters, C.; Poot, M.; Laudy, J.; et al. Congenital diaphragmatic hernia associated with duplication of 11q23-qter. Am. J. Med. Genet. A 2006, 140, 1580-1586. [CrossRef]

25. Rosenfeld, J.A.; Lacassie, Y.; El-Khechen, D.; Escobar, L.F.; Reggin, J.; Heuer, C.; Chen, E.; Jenkins, L.S.; Collins, A.T.; Zinner, S.; et al. New cases and refinement of the critical region in the 1q41q42 microdeletion syndrome. Eur. J. Med. Genet. 2011, 54, 42-49. [CrossRef]

26. Slavotinek, A.M.; Moshrefi, A.; Lopez Jiminez, N.; Chao, R.; Mendell, A.; Shaw, G.M.; Pennacchio, L.A.; Bates, M.D. Sequence variants in the HLX gene at chromosome 1q41-1q42 in patients with diaphragmatic hernia. Clin. Genet. 2009, 75, 429-439. [CrossRef] [PubMed]

27. Kantarci, S.; Ackerman, K.G.; Russell, M.K.; Longoni, M.; Sougnez, C.; Noonan, K.M.; Hatchwell, E.; Zhang, X.; Pieretti Vanmarcke, R.; Anyane-Yeboa, K.; et al. Characterization of the chromosome 1q41q42.12 region, and the candidate gene DISP1, in patients with CDH. Am. J. Med. Genet. A 2010, 152A, 2493-2504. [CrossRef] [PubMed] 
28. Casaccia, G.; Mobili, L.; Braguglia, A.; Santoro, F.; Bagolan, P. Distal 4p microdeletion in a case of Wolf-Hirschhorn syndrome with congenital diaphragmatic hernia. Birth. Defects Res. A Clin. Mol. Teratol. 2006, 76, 210-213. [CrossRef] [PubMed]

29. Gofin, Y.; Mackay, L.P.; Machol, K.; Keswani, S.; Potocki, L.; Di Gregorio, E.; Naretto, V.G.; Brusco, A.; Hernandez-Garcia, A.; Scott, D.A. Evidence that FGFRL1 contributes to congenital diaphragmatic hernia development in humans. Am. J. Med. Genet. A 2021, 185, 836-840. [CrossRef] [PubMed]

30. Tautz, J.; Veenma, D.; Eussen, B.; Joosen, L.; Poddighe, P.; Tibboel, D.; de Klein, A.; Schaible, T. Congenital diaphragmatic hernia and a complex heart defect in association with Wolf-Hirschhorn syndrome. Am. J. Med. Genet. A 2010, 152A, $2891-2894$. [CrossRef]

31. Unolt, M.; DiCairano, L.; Schlechtweg, K.; Barry, J.; Howell, L.; Kasperski, S.; Nance, M.; Adzick, N.S.; Zackai, E.H.; McDonaldMcGinn, D.M. Congenital diaphragmatic hernia in 22q11.2 deletion syndrome. Am. J. Med. Genet. A 2017, 173, 135-142. [CrossRef]

32. Wat, M.J.; Veenma, D.; Hogue, J.; Holder, A.M.; Yu, Z.; Wat, J.J.; Hanchard, N.; Shchelochkov, O.A.; Fernandes, C.J.; Johnson, A.; et al. Genomic alterations that contribute to the development of isolated and non-isolated congenital diaphragmatic hernia. J. Med. Genet. 2011, 48, 299-307. [CrossRef]

33. Machado, I.N.; Heinrich, J.K.; Barini, R.; Peralta, C.F. Copy number imbalances detected with a BAC-based array comparative genomic hybridization platform in congenital diaphragmatic hernia fetuses. Genet. Mol. Res. 2011, 10, 261-267. [CrossRef]

34. Otake, K.; Uchida, K.; Inoue, M.; Koike, Y.; Matsushita, K.; Miki, C.; Sugiyama, T.; Kusunoki, M. Congenital diaphragmatic hernia with a pure duplication of chromosome 1q: Report of the first surviving case. Pediatr. Surg. Int. 2009, 25, 827-831. [CrossRef] [PubMed]

35. Bermudez-Wagner, K.; Jeng, L.J.; Slavotinek, A.M.; Sanford, E.F. 2p16.3 microdeletion with partial deletion of the neurexin-1 gene in a female with developmental delays, short stature, and a congenital diaphragmatic hernia. Clin. Dysmorphol. 2013, $22,22-24$. [CrossRef]

36. Itsara, A.; Wu, H.; Smith, J.D.; Nickerson, D.A.; Romieu, I.; London, S.J.; Eichler, E.E. de novo rates and selection of large copy number variation. Genome Res. 2010, 20, 1469-1481. [CrossRef] [PubMed]

37. Conrad, D.F.; Pinto, D.; Redon, R.; Feuk, L.; Gokcumen, O.; Zhang, Y.; Aerts, J.; Andrews, T.D.; Barnes, C.; Campbell, P.; et al. Origins and functional impact of copy number variation in the human genome. Nature 2010, 464, 704-712. [CrossRef]

38. Urban, Z.; Hucthagowder, V.; Schürmann, N.; Todorovic, V.; Zilberberg, L.; Choi, J.; Sens, C.; Brown, C.W.; Clark, R.D.; Holland, K.E.; et al. Mutations in LTBP4 cause a syndrome of impaired pulmonary, gastrointestinal, genitourinary, musculoskeletal, and dermal development. Am. J. Hum. Genet. 2009, 85, 593-605. [CrossRef]

39. Hosokawa, S.; Takahashi, N.; Kitajima, H.; Nakayama, M.; Kosaki, K.; Okamoto, N. Brachmann-de Lange syndrome with congenital diaphragmatic hernia and NIPBL gene mutation. Congenit. Anom. 2010, 50, 129-132. [CrossRef] [PubMed]

40. Hague, J.; Twiss, P.; Mead, Z.; Park, S.M. Clinical Diagnosis of Classical Cornelia de Lange Syndrome Made from Postmortem Examination of Second Trimester Fetus with Novel NIPBL Pathogenic Variant. Pediatr. Dev. Pathol. 2019, 22, 475-479. [CrossRef]

41. Twigg, S.R.; Kan, R.; Babbs, C.; Bochukova, E.G.; Robertson, S.P.; Wall, S.A.; Morriss-Kay, G.M.; Wilkie, A.O. Mutations of ephrin-B1 (EFNB1), a marker of tissue boundary formation, cause craniofrontonasal syndrome. Proc. Natl. Acad. Sci. USA 2004, 101, 8652-8657. [CrossRef] [PubMed]

42. Smigiel, R.; Jakubiak, A.; Lombardi, M.P.; Jaworski, W.; Slezak, R.; Patkowski, D.; Hennekam, R.C. Co-occurrence of severe Goltz-Gorlin syndrome and pentalogy of Cantrell—Case report and review of the literature. Am. J. Med. Genet. A 2011, 155A, 1102-1105. [CrossRef]

43. Li, Y.; Bögershausen, N.; Alanay, Y.; Simsek Kiper, P.O.; Plume, N.; Keupp, K.; Pohl, E.; Pawlik, B.; Rachwalski, M.; Milz, E.; et al. A mutation screen in patients with Kabuki syndrome. Hum. Genet. 2011, 130, 715-724. [CrossRef]

44. Zarate, Y.A.; Zhan, H.; Jones, J.R. Infrequent Manifestations of Kabuki Syndrome in a Patient with Novel MLL2 Mutation. Mol. Syndromol. 2012, 3, 180-184. [CrossRef]

45. Srour, M.; Chitayat, D.; Caron, V.; Chassaing, N.; Bitoun, P.; Patry, L.; Cordier, M.P.; Capo-Chichi, J.M.; Francannet, C.; Calvas, P.; et al. Recessive and dominant mutations in retinoic acid receptor beta in cases with microphthalmia and diaphragmatic hernia. Am. J. Hum. Genet. 2013, 93, 765-772. [CrossRef] [PubMed]

46. Wilmink, F.A.; Papatsonis, D.N.; Grijseels, E.W.; Wessels, M.W. Cornelia de lange syndrome: A recognizable fetal phenotype. Fetal Diagn. Ther. 2009, 26, 50-53. [CrossRef] [PubMed]

47. Banait, N.; Fenton, A.; Splitt, M. Cornelia de Lange syndrome due to mosaic NIPBL mutation: Antenatal presentation with sacrococcygeal teratoma. BMJ Case Rep. 2015, 2015, bcr2015211006. [CrossRef] [PubMed]

48. Sweeney, N.M.; Nahas, S.A.; Chowdhury, S.; Campo, M.D.; Jones, M.C.; Dimmock, D.P.; Kingsmore, S.F.; RCIGM Investigators. The case for early use of rapid whole-genome sequencing in management of critically ill infants: Late diagnosis of Coffin-Siris syndrome in an infant with left congenital diaphragmatic hernia, congenital heart disease, and recurrent infections. Cold Spring Harb. Mol. Case Stud. 2018, 4, a002469. [CrossRef]

49. Wang, X.; Charng, W.L.; Chen, C.A.; Rosenfeld, J.A.; Al Shamsi, A.; Al-Gazali, L.; McGuire, M.; Mew, N.A.; Arnold, G.L.; Qu, C.; et al. Germline mutations in ABL1 cause an autosomal dominant syndrome characterized by congenital heart defects and skeletal malformations. Nat. Genet. 2017, 613-617. [CrossRef] [PubMed]

50. Bulfamante, G.; Gana, S.; Avagliano, L.; Fabietti, I.; Gentilin, B.; Lalatta, F. Congenital diaphragmatic hernia as prenatal presentation of Apert syndrome. Prenat. Diagn. 2011, 31, 910-911. [CrossRef] 
51. Suri, M.; Kelehan, P.; O'neill, D.; Vadeyar, S.; Grant, J.; Ahmed, S.F.; Tolmie, J.; McCann, E.; Lam, W.; Smith, S.; et al. WT1 mutations in Meacham syndrome suggest a coelomic mesothelial origin of the cardiac and diaphragmatic malformations. Am. J. Med. Genet. A 2007, 143A, 2312-2320. [CrossRef]

52. Cho, H.Y.; Lee, B.S.; Kang, C.H.; Kim, W.H.; Ha, I.S.; Cheong, H.I.; Choi, Y. Hydrothorax in a patient with Denys-Drash syndrome associated with a diaphragmatic defect. Pediatr. Nephrol. 2006, 21, 1909-1912. [CrossRef]

53. McVeigh, T.P.; Banka, S.; Reardon, W. Kabuki syndrome: Expanding the phenotype to include microphthalmia and anophthalmia. Clin. Dysmorphol. 2015, 24, 135-139. [CrossRef]

54. Revencu, N.; Quenum, G.; Detaille, T.; Verellen, G.; De Paepe, A.; Verellen-Dumoulin, C. Congenital diaphragmatic eventration and bilateral uretero-hydronephrosis in a patient with neonatal Marfan syndrome caused by a mutation in exon 25 of the FBN1 gene and review of the literature. Eur. J. Pediatr. 2004, 163, 33-37. [CrossRef]

55. Benjamin, D.R.; Juul, S.; Siebert, J.R. Congenital posterolateral diaphragmatic hernia: Associated malformations. J. Pediatr. Surg. 1988, 23, 899-903. [CrossRef]

56. Yu, L.; Wynn, J.; Cheung, Y.H.; Shen, Y.; Mychaliska, G.B.; Crombleholme, T.M.; Azarow, K.S.; Lim, F.Y.; Chung, D.H.; Potoka, D.; et al. Variants in GATA4 are a rare cause of familial and sporadic congenital diaphragmatic hernia. Hum. Genet. 2013, 132, 285-292. [CrossRef] [PubMed]

57. Kammoun, M.; Souche, E.; Brady, P.; Ding, J.; Cosemans, N.; Gratacos, E.; Devriendt, K.; Eixarch, E.; Deprest, J.; Vermeesch, J.R. Genetic profile of isolated congenital diaphragmatic hernia revealed by targeted next-generation sequencing. Prenat. Diagn. 2018, 38, 654-663. [CrossRef]

58. Jay, P.Y.; Bielinska, M.; Erlich, J.M.; Mannisto, S.; Pu, W.T.; Heikinheimo, M.; Wilson, D.B. Impaired mesenchymal cell function in Gata4 mutant mice leads to diaphragmatic hernias and primary lung defects. Dev. Biol. 2007, 301, 602-614. [CrossRef]

59. Goumy, C.; Gouas, L.; Marceau, G.; Coste, K.; Veronese, L.; Gallot, D.; Sapin, V.; Vago, P.; Tchirkov, A. Retinoid pathway and congenital diaphragmatic hernia: Hypothesis from the analysis of chromosomal abnormalities. Fetal Diagn. Ther. 2010, 28, 129-139. [CrossRef] [PubMed]

60. You, L.R.; Lin, F.J.; Lee, C.T.; DeMayo, F.J.; Tsai, M.J.; Tsai, S.Y. Suppression of Notch signalling by the COUP-TFII transcription factor regulates vein identity. Nature 2005, 435, 98-104. [CrossRef] [PubMed]

61. Rossetti, L.Z.; Glinton, K.; Yuan, B.; Liu, P.; Pillai, N.; Mizerik, E.; Magoulas, P.; Rosenfeld, J.A.; Karaviti, L.; Sutton, V.R.; et al. Review of the phenotypic spectrum associated with haploinsufficiency of MYRF. Am. J. Med. Genet. A 2019, 179, 1376-1382. [CrossRef]

62. Pinz, H.; Pyle, L.C.; Li, D.; Izumi, K.; Skraban, C.; Tarpinian, J.; Braddock, S.R.; Telegrafi, A.; Monaghan, K.G.; Zackai, E.; et al. de novo variants in Myelin regulatory factor (MYRF) as candidates of a new syndrome of cardiac and urogenital anomalies. Am. J. Med. Genet. A 2018, 176, 969-972. [CrossRef] [PubMed]

63. Jin, S.C.; Homsy, J.; Zaidi, S.; Lu, Q.; Morton, S.; DePalma, S.R.; Zeng, X.; Qi, H.; Chang, W.; Sierant, M.C.; et al. Contribution of rare inherited and de novo variants in 2871 congenital heart disease probands. Nat. Genet. 2017, 49, 1593-1601. [CrossRef] [PubMed]

64. Reis, L.M.; Tyler, R.C.; Schilter, K.F.; Abdul-Rahman, O.; Innis, J.W.; Kozel, B.A.; Schneider, A.S.; Bardakjian, T.M.; Lose, E.J.; Martin, D.M.; et al. BMP4 loss-of-function mutations in developmental eye disorders including SHORT syndrome. Hum. Genet. 2011, 130, 495-504. [CrossRef]

65. Bashamboo, A.; Eozenou, C.; Jorgensen, A.; Bignon-Topalovic, J.; Siffroi, J.P.; Hyon, C.; Tar, A.; Nagy, P.; Sólyom, J.; Halász, Z.; et al. Loss of Function of the Nuclear Receptor NR2F2, Encoding COUP-TF2, Causes Testis Development and Cardiac Defects in 46,XX Children. Am. J. Hum. Genet. 2018, 102, 487-493. [CrossRef]

66. Tuzovic, L.; Yu, L.; Zeng, W.; Li, X.; Lu, H.; Lu, H.M.; Gonzalez, K.D.; Chung, W.K. A human de novo mutation in MYH10 phenocopies the loss of function mutation in mice. Rare. Dis. 2013, 1, e26144. [CrossRef]

67. Yu, L.; Bennett, J.T.; Wynn, J.; Carvill, G.L.; Cheung, Y.H.; Shen, Y.; Mychaliska, G.B.; Azarow, K.S.; Crombleholme, T.M.; Chung, D.H.; et al. Whole exome sequencing identifies de novo mutations in GATA6 associated with congenital diaphragmatic hernia. J. Med. Genet. 2014, 51, 197-202. [CrossRef]

68. Ackerman, K.G.; Herron, B.J.; Vargas, S.O.; Huang, H.; Tevosian, S.G.; Kochilas, L.; Rao, C.; Pober, B.R.; Babiuk, R.P.; Epstein, J.A.; et al. Fog2 is required for normal diaphragm and lung development in mice and humans. PLoS Genet. 2005, 1, e10. [CrossRef] [PubMed]

69. de Ligt, J.; Veltman, J.A.; Vissers, L.E. Point mutations as a source of de novo genetic disease. Curr. Opin. Genet. Dev. 2013, 23, 257-263. [CrossRef]

70. Deciphering Developmental Disorders Study. Prevalence and architecture of de novo mutations in developmental disorders. Nature 2017, 542, 433-438. [CrossRef] 